\title{
DRUG RESISTANCE IN LEPROSY-MYTH OR MENACE?
}

The papers that comprise this issue of Leprosy Review will provide sombre reading for field workers and Ministries of Health alike. They bring together within a convenient compass a mass of factual and experimental evidence that must affect the whole strategy of leprosy control throughout the world, with its implications for finance and staff, for training programmes and the integration of leprosy treatment/control schemes into the general health services. Furthermore, the findings here set forth will necessarily affect the fund-raising and propaganda activities of voluntary agencies.

For practical purposes, these papers are concerned with bacilli resistant to dapsone, but cross-resistance between dapsone and the sulphonamides, and between thiambutosine and thiacetazone provides interesting (though less important) clinical and experimental data.

Ever since doctors and medical auxiliaries dared to try to treat with a single drug patients suffering from multibacillary forms of the chronic mycobacterial infection that is leprosy, they were really asking for this to happen, in the light of experience painfully acquired over the years in many countries with the sister-disease, tuberculosis.

The only surprising feature in this sad story is the time factor- 20 years were to elapse after the initiation of an essentially monotherapeutic treatment before the first cloud, "no bigger than a man's hand", appeared on the Malaysian horizon, and then another dozen years or so before the real threat of the emergence of drug-resistance on a wide scale became apparent. Workers in the early days of the sulphone era may perhaps be forgiven for their optimistic assumptions. After all, the introduction of the sulphones did mark the dawn of a new day for leprosy sufferers, especially in the African continent. Leprosy did seem to be different in many respects from infections with related organisms, and the sulphones in extremely low serum concentrations seemed to be mycobacteriostatic.

Having been privileged to examine clinically the first patients (at Sungei Buloh, Selangor, Malaysia) whose relapse, it was reasonably suspected, was due to the emergence of sulphone-resistant organisms, and having seen the microscopical evidence in smears containing numerous morphologically normal organisms, the writer was early alerted to the possibility that this initial observation might be the precursor of many more. By that time (1963), clinical suspicions could be confirmed by the elegant mouse foot-pad technique brilliantly adapted to demonstrate the stepwise development of resistance. The rest is history.

The 5th Expert Leprosy Committee of the World Health Organization meeting October 1976 (whose Report should be appearing shortly), examined the evidence accruing from many sources of the emergence of dapsone-resistant Mycobacterium leprae and of the apparent appearance of resistance in wild strains (indicating some kind of decrease of susceptibility to drug concentrations, formerly mycobacteriostatic) isolated from newly-diagnosed patients, and made recommendations for therapeutic regimens that would, it was hoped, postpone indefinitely the emergence of such forms on an unmanageable scale and treat 
successfully those patients whose clinical relapse is due to drug-resistant bacilli. Meanwhile, the consequences of these findings have to be accepted by those responsible for leprosy treatment/control programmes, and steps taken urgently to forestall the imminent threat of a pandemic of patients with drug-resistant bacilli.

One curious observation is the patchy reporting of such cases. Much depends, naturally, on the length of time that the sulphones have been used in any given area and the lepromatous/tuberculoid (or, better, the multibacillary/ paucibacillary) ratio, which may be as low as $1: 10$ or even 1:20 in some African countries where regular whole-population examinations are done. Much more depends on the degree of awareness or suspicion shown by doctors and medical auxiliaries. Ignorance of the clinical presentations of skin lesions due to drug-resistant bacilli, misdiagnosis of clinical and bacteriological relapse (as erythema nodosum leprosum), and a failure to use the investigative laboratory procedures that are (or should be) generally available in field-work would account for the non-recognition of such cases. It cannot be too strongly emphasized that bacteriological relapse frequently precedes clinical evidence of relapse: therefore regular and frequent slit-smear examinations should be performed on patients whose multibacillary leprosy is apparently quiescent after adequate periods of treatment.

For most countries, clinical confirmation of clinical suspicion of the emergence of resistance will be the norm, with field laboratory work of the highest possible standard-for the recognition and enumeration of "solid staining bacilli". The experimental confirmation by the mouse foot-pad technique is beyond the reach-or the financial and operational resources-of the majority of countries where the problem is certainly occurring now. It is here that offers of international co-operation would be most welcome-Japan to South-East Asian countries, United States of America to Central and South America, England and Belgium to Africa. Noblesse oblige when the threat is global. Typical cases could be selected for laboratory confirmation-as a convincing demonstration of the actual occurrence of relapse due to resistant bacilli.

\section{Treatment}

Fortunately, the great majority of leprosy sufferers in the world may still be treated with a single drug-the cheap and effective dapsone. The more intensely case-finding surveys are done, the greater the proportion of cases of paucibacillary and self-healing leprosy that will be detected. In these, cell-mediated immunity will suffice, with a single drug, to overcome the infection, and the risk of the emergence of dapsone resistant bacilli is negligible. Perhaps a greater use could be made of the lepromin reaction in those cases of indeterminate leprosy that may really be pre-lepromatous. A persistently negative Mitsuda reaction would ideally indicate the need for prolonged, multidrug therapy.

It is in those patients suffering from multibacillary forms of leprosy that real therapeutic and financial difficulties will arise, and problems associated with controversial public relations aspects. In theory, more than one drug should be given: which drugs and for how long? are questions that may evoke different answers in different countries. Dapsone is a sine qua non. In addition, rifampicin for a few weeks at a dose of $600 \mathrm{mg}$ a day, or at a higher dose $(900 \mathrm{mg})$ on 2 successive days every month for some months, or even a single dose of 
$1500 \mathrm{mg}$-all regimens are on trial. Dapsone is given concurrently and then continued alone.

Clofazimine has its advocates, given at a dose of $100 \mathrm{mg}$ every other day (with daily dapsone) for 3-6 months, to be followed by dapsone alone. Other drugs, such as thiacetazone and ethionamide, will have to be investigated further in this context. The general recommendation to continue treatment "for life" for patients with multibacillary forms of leprosy, itself contains the seeds of resistant bacillary forms, since a small but definite proportion of persister organisms-once they leave the dormant stage and begin multiplying again-will be potentially resistant mutants.

Whether we like it or not, we must assume that for some time to come-because of expense and the logistic difficulties of implementing multi-drug regimens-many countries will continue to favour monotherapy with dapsone for all patients suffering from leprosy whatever the form of the disease.

Ideally, and in order to forestall-or indefinitely postpone-the emergence of dapsone-resistant bacilli, many leprologists now recommend that a high daily dose of dapsone be given from the outset of treatment to all patients suffering from leprosy. Herein lies a snag. It is common experience that a high dose of dapsone, administered from day one to all patients suffering from multibacillary forms of leprosy, will be followed within a few days in many patients by the lesions of erythema nodosum leprosum or, in the case of borderline-lepromatous leprosy, by the signs of polyneuritis (reversal reaction). In some countries, this proportion has been so high as to imperil the acceptance of the leprosy programme. Our French colleagues have done much work on what they call the "reactogenic" properties of the different drugs used for leprosy. Before such findings are dismissed as "anecdotal" or "uncontrolled", we should do well to remind ourselves of the considerable variations in the clinical pattern of leprosy in different countries and the differences in response to anti-leprotics. Insomnia and manic hyperactivity not infrequently follows every dose of $100 \mathrm{mg}$ dapsone in some individuals. Fixed eruption following sulphone therapy may vary between $0.1 \%$ and $3.0 \%$ in different communities, and dermal fibrosis may vary from the negligible to the enormous; some communities show unduly high prevalence rates of kebid, ainhum, juxta-articular nodules, paratrochanteric fibrosis, and palmar and plantar hyperkeratosis, framboeisial or following friction. Some patients with leprosy seem especially prone to the rapid development of intraneural fibrosis and a dense fibrous sheath around peripheral nerve trunks that may even be the site of deposit of calcium salts. However, the local situation, including the ready availability of facilities for diagnosing and treating such episodes-however precipitated-must determine the posology of anti-leprotics, and the immediate risks involved must be nicely balanced against the remoter benefits (to the patient himself and the community) of the relegation to the distant future of the emergence of dapsone-resistant bacilli.

The treatment of patients with reasonably ascertained dapsone-resistant disease has hitherto been simple-monotherapy with either rifampicin or clofazimine. But, by the same token, multidrug therapy (with both rifampicin and clofazimine) must henceforth be recommended, especially since rifampicinresistant bacilli have

to these cripplingly costly proposals?

The question is far from academic or theoretical in the light of the discovery that some recently diagnosed patients are suffering from multibacillary forms of 
leprosy attributable to bacilli primarily resistant to sulphones. And if such cases exist now (suggestive of the pathogenicity and invasiveness of these organisms), it will not be long before, in the same community exposed to the same bacilli, some people will succumb to forms of leprosy which, by reason of innate degrees of cell-mediated immunity, will declare themselves as "tuberculoid" leprosy. The possibility that such cases are in reality infected with dapsone-resistant bacilli will doubtless be overlooked until lack of the expected response to monotherapy with dapsone alerts the clinician.

The implications of all these serious observations for governments and voluntary agencies will not be lost. The future of public relations regarding leprosy bristles with difficulties; awkward questions will be asked about published claims for rapid and cheap "cure", about the real extent of the menace of drug resistance and the cost of available antileprotic drugs: and patient resistance may be matched by official disillusionment and resignation to the "inevitable".

Another implication concerns the desirability and practicability of integrating leprosy programmes with the general health services: since the antileprosy campaign is likely to prove more difficult and more protracted (and more expensive) than hitherto imagined, Ministries of Health will still need to be able to call upon expert advice at all levels. The delicate balance between the advantages to be gained by integrating leprosy into the general health programme will have to be examined against the risk of perpetuating the stigma of the disease and incurring the expense of organizing separate services for several diseases.

This whole question of drug-resistance in leprosy underlines the urgent necessity for developing new anti-leprotic agents. Perhaps some derivatives of hydnocarpic acid may show the way forward in attacking the multiplying organism at a novel and vulnerable site.

It also implies that the whole question of infection with Mycobacterium leprae must be taken much more seriously than it has been. Clinical standards and laboratory cover must likewise be raised, and more resources made available by all possible means so that we may do what we can while we can to control this most challenging of diseases.

S. G. BROWNE 